\title{
Leistungsentgelt in den Kommunen: Praxis einer umstrittenen Regelung
}

Leistungsentgelt gilt der Vereinigung der kommunalen Arbeitgeberverbände als dringend erforderlich, um endlich auch im öffentlichen Dienst dem Leistungsgedanken zum Durchbruch zu verhelfen und die Modernisierung voranzutreiben. Viele Gewerkschafter halten Leistungsentgelt im öffentlichen Dienst hingegen für eine unnötige und ineffektive Fehlanpassung an die Privatwirtschaft, auf die im Interesse an Daseinsvorsorge orientierter öffentlicher Dienstleistungen verzichtet werden sollte. An einem gesicherten Erfahrungs- und Wissensbestand über die praktische Umsetzung und die Wirkungen fehlte es jedoch bisher.

WERNER SCHMIDT, ANDREA MÜLLER

\section{Problemhintergrund und Forschungshypothesen}

Mit dem Tarifvertrag für den öffentlichen Dienst (TVöD) wurde Leistungsentgelt oder leistungsorientierte Bezahlung (LOB) für alle Tarifbeschäftigten des Bundes und der Kommunen eingeführt. Damit sollte ein Wechsel vom sogenannten Alimentationsprinzip zum Prinzip der Leistungsorientierung eingeleitet werden. Die Orts- und Familienzuschläge des Bundesangestelltentarifvertrags (BAT) wurden durch leistungsabhängige Entgeltbestandteile ersetzt. Seit dem Jahr 2007 können die Kommunen, mit denen wir uns hier ausschließlich beschäftigen werden, leistungsabhängig bezahlen, soweit sich Arbeitgeber und Personalräte auf den Abschluss einer einvernehmlichen Dienstvereinbarung (DV) verständigen. Anfangs sah der Tarifvertrag für den öffentlichen Dienst (TVöD) dafür ein Budget im Umfang von $1 \%$ der ständigen Monatsentgelte des Vorjahres vor, inzwischen wurde dieses auf Drängen der Vereinigung der kommunalen Arbeitgeberverbände (VKA) auf $2 \%$ angehoben. Über die weitere Entwicklung dürfte in der Tarifrunde 2014 entschieden werden.

Der $\$ 18$ TVöD, der die einschlägigen Bestimmungen enthält, nennt als Ziele von LOB die „Verbesserung der öffentlichen Dienstleistungen“, „Motivation“, „Eigenverantwortung “ und „Führungskompetenz“. Zudem sollen in der abzuschließenden einvernehmlichen Dienstvereinbarung
„Ziele zur Sicherung und Verbesserung der Effektivität und Effizienz, insbesondere für Mehrwertsteigerungen (z. B. Verbesserung der Wirtschaftlichkeit, der Dienstleistungsqualität, der Kunden-/Bürgerorientierung)“ festgelegt werden. Die VKA verspricht sich von LOB neben der klassischen Anreizfunktion nicht zuletzt eine Verbesserung der als mangelhaft geltenden Führungskultur. Bei ver.di werden die Regelungen zum Leistungsentgelt überwiegend als wirkungslos oder als Versuch weiterer Arbeitsverdichtung abgelehnt. Der Regelung zugestimmt wurde von ver.di lediglich, weil der TVöD und der Flächentarifvertrag gefährdet schienen. Insgesamt erfolgte die Einführung leistungsorientierter Bezahlung im öffentlichen Dienst nicht auf Basis eines nachhaltigen tarifpolitischen Konsenses.

Die Bedingung, dass eine einvernehmliche Dienstvereinbarung abgeschlossen werden muss, führt dazu, dass bei Widerstand einer Betriebspartei oder beiderseitigem Desinteresse auf eine LOB-Einführung verzichtet werden kann. Allerdings darf ohne DV nur ein Teil des Prämienbudgets ausgeschüttet werden. ${ }^{1}$ Als Methoden der Leistungsbewertung sieht der TVöD Zielvereinbarungen (ZV), die

1 Ausgeschüttet werden sollen ohne DV lediglich $6 \%$ des Septemberentgelts, d. h. deutlich weniger als das aktuelle Prämienbudget von $2 \%$ des Vorjahresentgelts. Der Rest soll bis zu einem DV-Abschluss thesauriert werden (vgl. auch Dannenberg 2013). 
sogenannte Systematische Leistungsbewertung (SLB) oder eine Kombination beider Verfahren vor. Ver.di empfiehlt im Falle der LOB-Einführung, Zielvereinbarungen als Grundlage der Leistungsbewertung zu nutzen, weil diese den Beschäftigten Beteiligungsmöglichkeiten eröffnen (Dürk/ Sternatz 2007; Herbing 2004). Die VKA empfiehlt eher die Kombinationsvariante, die sich hin zu ZV entwickeln könne.

Der Umstand, dass leistungsbezogene Entlohnung im deutschen öffentlichen Dienst neu ist, die vorgesehene dezentrale Umsetzungspraxis, die strittige Haltung der Tarifparteien sowie noch zu skizzierende widersprüchliche wissenschaftliche Befunde zur Wirksamkeit von leistungsbezogenen Entlohnungssystemen werfen mit Blick auf die Einführung leistungsorientierter Bezahlung eine Reihe von Forschungsfragen auf und sprechen für nachfolgende forschungsleitende Hypothesen:

(1) Zunächst bleibt angesichts der tarifvertraglichen Verpflichtung, dass LOB nur auf Basis einer einvernehmlichen Dienstvereinbarung eingeführt werden darf, völlig offen, in wie vielen Fällen es gelingt, diese grundlegende Bedingung zu erfüllen und wie häufig folglich LOB in den Kommunen überhaupt eingeführt wird. Diese Unsicherheit besteht umso mehr, weil frühere Versuche, Leistungsentgelt in den Kommunen auf freiwilliger Basis zu etablieren, wenig erfolgreich waren (Tondorf/Jochmann-Döll 2004). Allerdings ist in Rechnung zu stellen, dass die vorgegebene reduzierte Ausschüttung bei fehlender DV einem Umsetzungsverzicht entgegenwirkt. Eine Befragung von Personalräten erbrachte, dass $23 \%$ der Kommunen in NordrheinWestfalen (NRW) auf Leistungsdifferenzierung verzichten (Schmidt et al. 2011a, S. 108). Da jedoch in NRW größere Kommunen dominieren und der dortige Arbeitgeberverband als besonders LOB-affin einzuschätzen war, war bundesweit mit einer etwas geringeren Umsetzung von LOB zu rechnen.

(2) Im Falle der Einführung eines Leistungsentgeltsystems war zu erwarten, dass die Mehrheit der Kommunen die Systematische Leistungsbewertung (SLB) einführt und im Sinne eines unilateralen Beurteilungsverfahrens anwendet. ${ }^{2}$ Hierfür sprachen auch Erfahrungen aus der Industrie, wo Zielvereinbarungen, als alternative Methode der Leistungsbewertung, meist nur eine äußerst geringe Rolle spielen. ${ }^{3}$ Zudem lag die Annahme nahe, dass der als weniger aufwendig geltenden SLB dort der Vorzug gegeben wird, wo Verantwortliche sich ohnehin wenig Effekte von LOB versprechen und deshalb primär darauf setzen, den hiermit verbundenen Aufwand gering zu halten (Keller 2010, S. 106). Andererseits konnte keineswegs ausgeschlossen werden, dass Zielvereinbarungen (ZV) in den Kommunen häufiger als in der Industrie die präferierte Methode der leistungsorientierten Bezahlung sein würden, zumal sie in einigen Dienstleistungsunternehmen bereits etabliert (Fischer 2010) und die von ver.di empfohlene Methode sind.

(3) Der Nutzen „leistungsorientierter Bezahlung“ ist nicht nur zwischen ver.di und der VKA umstritten, uneins ist sich auch die diesbezügliche Forschung. Für einen Teil der Personalökonomen gelten monetäre Anreize nicht nur als „essence of economics“, sondern auch als „an essential part of organizational design“ (Lazear/Gibbs 2009, S. 285). $\mathrm{Da}$ anzunehmen ist, dass die Interessen der Beschäftigten nicht per se mit denen der Organisation identisch sind (Sadowski et al. 1999), sei ohne besondere Anreize mit opportunistischem Verhalten, Bummelei etc. zu rechnen (Principal-Agent-Problem). Werden Bewertungskriterien oder Ziele jedoch falsch gesetzt, so muss auch aus der Sicht eines an Nutzenmaximierung orientierten Konzeptes mit Fehlsteuerungen und ausbleibenden oder kontraproduktiven Effekten gerechnet werden.

(4) Ebenfalls mit Negativeffekten muss unter der Voraussetzung nutzenmaximierenden Verhaltens gerechnet werden, wenn Beschäftigte um eine begrenzte Menge positive Bewertungen (vorgegebene Bewertungsverteilung, z. B. durch Quotierung) oder ein feststehendes Prämienbudget konkurrieren müssen, denn sie werden dann nicht zur Kooperation angeregt (Lazear/Gibbs 2009, S. 301). Das gemäß $\S 18$ TVöD fixe Budget lässt somit zunehmendes Konkurrenzverhalten unter den Beschäftigten erwarten. Darüber hinaus fehlt es an einem eindeutigen Zusammenhang zwischen Leistungsverhalten und Prämienerhalt. Unsicherheit der Beschäftigten über die monetären Folgen des Leistungsverhaltens ist die Folge.

(5) Ein eher psychologisch orientierter Teil der Literatur (Cognitive Evaluation Theory bzw. Self Determination Theory) betrachtet Leistungsanreize hingegen mit grundsätzlicher Skepsis. Argumentiert wird, dass ,intrinsische“, also aus der Arbeit selbst bezogene Motivation durch extrinsische Anreize Schaden nehmen könne (Deci 1971, 1976; Frey 1997; Ryan/Deci 2000; Sansone/Harackiewicz 2000). Äußere Anreize können intrinsische Motivation verdrängen, wenn die Anreize als kontrollierend wahrgenommen werden und Selbstbestimmung und Selbstachtung darunter leiden. Werden Anreize hingegen als „supportive" empfunden, so können sie sich auch unterstützend auf die intrinsische Motivation auswirken (Frey/Jegen 2001, S. 594f.).

(6) Prinzipiell kann ein Verlust intrinsischer Motivation durch externe Anreize kompensiert werden, soweit Letztere hinreichend hoch ausfallen (Matiaske/Weller 2008). Je schwächer die intrinsische Motivation, desto kostengünstiger wären demnach Anreizeffekte durch Leistungsentgelt zu erzielen. Nun besteht im öffentlichen Dienst allerdings auch der Umstand, dass das LOB-Budget im Branchenvergleich ausgesprochen gering ist und $\mathrm{zu}-$

2 Ver.di befürchtete, dass häufig die als wenig effektiv geltende Regelbeurteilung zum Vorbild genommen wird.

3 Gesamtmetall (2013,Tab. 8) weist für 2012 1,5\% der Beschäftigten mit ZV aus (vgl. auch Bahnmüller/Schmidt 2009, S. 246) 
gleich die Interessendivergenz zwischen Organisation und Beschäftigten als wenig ausgeprägt gelten darf. Wegen des gemeinsamen Interesses von kommunalen Arbeitgebern und Beschäftigten an der Erbringung öffentlicher Dienstleistungen (Daseinsvorsorge) kann nicht ausgeschlossen werden, dass eine besondere, mit hohen intrinsischen Anteilen verbundene "public sector motivation“ existiert (Perry/Wise 1990; Perry 1996). Dies kann zur Folge haben, dass in dieser Konstellation nicht allein bei fehlerhafter LOB-Anwendung, sondern bei Etablierung von Leistungsentlohnung per se die vorhandene intrinsische Motivation unterhöhlt und der erwartete Motivationsanreiz in sein Gegenteil verkehrt wird.

(7) Insofern kann nicht überraschen, wenn Leistungsentgelt gerade für den öffentlichen Dienst oft als ein wenig geeignetes Entlohnungssystem gilt (bspw. Kessler/Purcell 1992; Brown et al. 2003; OECD 2005). Jedoch ist auch zu sehen, dass trotz vielfacher Einwände und Vorbehalte (Agrawal 2012) LOB im öffentlichen Dienst etlicher Länder seit Längerem praktiziert wird (Demmke 2009; Marsden 2010; Schmidt et al. 2011b). Vor diesem Hintergrund scheint mit Blick auf die Einführung von LOB im deutschen öffentlichen Dienst Skepsis zwar durchaus angebracht zu sein, jedoch ist nicht damit zu rechnen, dass sie sich als gänzlich funktionslos entpuppt.

(8) Darüber hinaus sind methodenspezifische Einflüsse wahrscheinlich. Bei SLB ist der Nachteil zu erwarten, dass Beurteilungen bekanntermaßen subjektiven Einschätzungen folgen, was alltagssprachlich meist als „Nasenfaktor" bezeichnet wird (vgl. auch Breisig 2003, S. 312). Erwartungen von Beschäftigten, die von einem kalkulierbaren Tausch „Leistung gegen Prämie“ ausgehen, werden dann enttäuscht, wenn Bewertungskriterien intransparent bleiben bzw. subjektiv geprägt sind. Insofern sind auch wir im Vorfeld unserer Untersuchung zur Umsetzung der leistungsorientierten Bezahlung im öffentlichen Dienst von der Annahme ausgegangen, dass die SLB, was Funktionalität und Akzeptanz anbelangt, größere Schwächen hat als die alternative Methode der Zielvereinbarung. Als weiterer Vorteil der Zielvereinbarungen gilt, dass sie bei methodengerechter Anwendung eine gewisse Mitwirkung der Beschäftigten bei der Gestaltung der Leistungsbedingungen ermöglichen (Kratzer/Nies 2009, S. 252f.).

(9) Als gesichertes Wissen gilt in der einschlägigen Literatur (Tondorf 2007, S. 48; Schettgen 1996, S. 264; Breisig 1998, S. 183) eine Tendenz zur besseren Bewertung von Beschäftigten mit höherem Status. Dieser „Hierarchieeffekt" dürfte in einem gewissen Zusammenhang mit der Subjektivität von Beurteilungsprozessen stehen und bei SLB häufiger auftreten. Ähnliches dürfte für die Benachteiligung von Frauen bei Leistungsbewertungen gelten (Tondorf/Jochmann-Döll 2005). Folgt man der Literatur, so ist durch LOB mit einer moderaten Zunahme von Entgeltspreizung und geschlechtsspezifischen Entlohnungsdifferenzen (gender pay gap) zu rechnen.

\section{Empirische Basis und Erhebungs- methoden}

Die empirischen Analysen, mit denen wir den skizzierten Forschungsfragen und Hypothesen nachgegangen sind, erfolgten im Rahmen einer von der Hans-Böckler-Stiftung geförderten Studie (Schmidt/Müller 2013). Um ein präzises und facettenreiches Bild zu zeichnen, wurden Interviews mit Arbeitgebervertretern und Personalräten sowie Gruppendiskussionen mit Führungskräften und Beschäftigten geführt. Zudem wurden im Jahr 2011 in einer Zufallsstichprobe von 1.781 Kommunen mit mehr als 3.000 Einwohnern sowohl die Arbeitgeber (Befragung AG) als auch die Personalräte (Befragung PR) schriftlich befragt. Mit einem kürzeren Fragebogen wurden darüber hinaus die Arbeitgeber kleinerer Kommunen in die Untersuchung einbezogen. ${ }^{4}$ Der Rücklauf betrug 426 bzw. 24 \% (AG), 604 bzw. 35 \% (PR) und 206 bzw. $33 \%$ (KL). Um einen möglichst großen Teil der Bruttostichprobe abzudecken, wurde für Basisangaben und andere "hard facts" aus den Daten der Erhebungen KL und AG, ergänzt um Fälle aus der PR-Erhebung (soweit für eine Kommune keine Arbeitgeberangaben vorliegen), ein integrierter Datensatz (FALL+) mit 1.064 Fällen gebildet. Soweit merkliche Einschätzungs- und Deutungsspielräume bestehen oder mit Interessengebundenheit zu rechnen ist, stützt sich die Argumentation jedoch auf die separaten Angaben der Akteure.

Schließlich haben wir in 34 Kommunen der AG/PRStichprobe auch Beschäftigte schriftlich befragt, wobei wir von 7.303 ausgegebenen Fragebogen 3.211 zurückerhielten (Befragung BESCH34).

\section{Ergebnisse}

\subsection{Verbreitung und Ausgestaltung}

Leistungsentgelt nach $₫ 18$ TVöD-VKA wird in $55 \%$ der Kommunen im Sinne einer auf einer Leistungsbewertung basierenden, differenzierten Ausschüttung von Prämien praktiziert. Der Anteil der von LOB abgedeckten Beschäftigten liegt bei $59 \%$. Die Umsetzung fällt damit etwas geringer aus, als wir es erwartet hatten. In den anderen Kommunen werden die dafür vorgesehenen Mittel pauschal an die Beschäftigten ausgeschüttet (oft nach der 6\%-Regel, meist jedoch der volle Betrag). Der Verzicht auf LOB begründet sich nicht allein in einer Verweigerung der Personalräte. $57 \%$ der Arbeitgeber (Befragung AG) ohne

4 Zusätzliche Bruttostichprobe von 628 Fällen mit weniger als 3.000 Einwohnern (Befragung KL). 
ABB.

\section{Leistungsorientierte Bezahlung (LOB) - Effekte aus Arbeitgebersicht}

Angaben in Prozent, sortiert nach Mittelwert

verbessert (1) —eher verbessert (2) —unverändert (3)

- eher verschlechtert (4) verschlechtert (5)

Welche Effekte hatte die Einführung der LOB auf nachfolgende Punkte?"*

Leistungsgerechte Bezahlung

Führungskompetenz

Eigenverantwortung

Kunden-/Bürgerorientierung

Dienstleistungsqualität

Motivation

Leistung

Führen mit Zielen

Beteiligung der Beschäftigten

Aufgaben- und Prozesssteuerung

Verhältnis Führungskräfte - Mitarbeiter

Effizienz der Arbeitsprozesse

Wirtschaftlichkeit

Personalkosteneffizienz

Verhältnis unter den Kolleg/inn/en

Begrenzung des Leistungsdrucks
*Arbeitgeber-Befragung: Datensatz AG. Quelle: Schmidt/Müller (2013, S. 109).

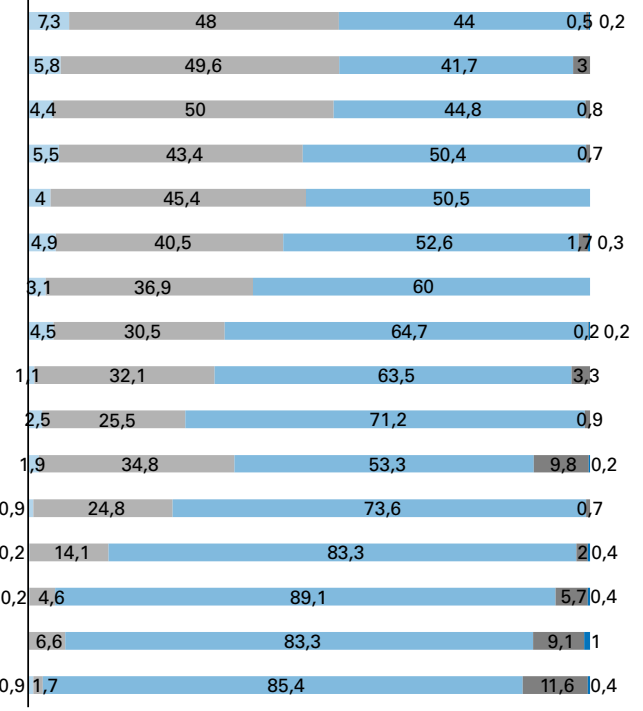

238

2,42

2,47 den Aufwand zu verzichten - auch wenn dies auf Kosten der Wirksamkeit geht. Zwar existiert in der Praxis durchaus eine gewisse Bandbreite und nicht selten orientieren sich Kommunen an den von Organisationsberatern aus dem Privatsektor entliehenen Empfehlungen. Doch 35 \% der Arbeitgeber mit SLB geben explizit an, die Bewertungskriterien seien „an die Kriterien der dienstlichen Regelbeurteilung angelehnt ${ }^{\text {“ }}{ }^{5}$ Dies ist eine Praxis, die so von den Tarifparteien erklärtermaßen nicht intendiert war.

\subsection{Funktionalität und Methode}

Was bewirkt Leistungsentgelt in den untersuchten Kommunen? Weniger als die Hälfte der Arbeitgeber berichtet von (eher schwachen) positiven Effekten auf Motivation und Leistung, immerhin $55 \%$ sind jedoch der Auffassung, die Bezahlung sei leistungsgerechter geworden. Etwas mehr als die Hälfte der Arbeitgeber sehen Führungskompetenz, Eigenverantwortung und Kunden-/Bürgerorientierung sowie (knapp die Hälfte) Dienstleistungsqualität (eher) gestärkt. Insgesamt stellen sich die Effekte aus Arbeitgeberperspektive in doppelter Weise als schwach dar: erstens, weil in vielen Kommunen Effekte gänzlich ausbleiben, zweitens, weil dort, wo Wirkungen grundsätzlich zu konstatieren sind, diese letztlich eher schwach bleiben (Abbildung 1). Der Mittelwert ist gleichwohl positiv.

Auch die Personalräte berichten mehrheitlich von ausbleibenden Effekten und sind sich darin mit den befragten Beschäftigten einig (Abbildung 2). Trotzdem gilt mit Abstrichen auch in der Befragung der Personalräte, dass die genannten Effekte zwar als ausgesprochen schwach, jedoch als im Mittel positiv wahrgenommen werden. ${ }^{6}$ Von den befragten Beschäftigten berichten $25 \%$ von positiven, $15 \%$ jedoch auch von negativen Effekten auf die Motivation. Separat danach gefragt, benennen auch $17 \%$ der Arbeitgeber und $34 \%$ der Personalräte das Problem der Demotivation. Ausgeprägt positiv wirkt sich die Einführung von Leistungsentgelt jedoch dahin gehend aus, dass es nunmehr zu Mitarbeitergesprächen kommt, was zuvor selten der Fall war (Schmidt/Müller 2013, S. 123).

Befürchtungen, LOB könnte vermittelt über individuelle Mehrleistung und Personaleinsparungen zu einem Sinken des kollektiven Entgeltvolumens beitragen und zu einer Verschlechterung der "terms of trade“ der Ware Arbeitskraft führen, haben sich im Großen und Ganzen nicht bewahrheitet. Tatsächlich scheint es sich eher umgekehrt zu verhalten: Nicht LOB-bedingte Mehrleistung eröffnet Möglichkeiten der Personalkostensenkung, sondern es sind vielmehr der Kombination von SLB und ZV eingesetzt, „in der die ZV Anteile überwiegen“. In den kleinen Kommunen (KL) kommen ZV noch in $11 \%$ der Fälle vor.

Zielvereinbarungen werden vielerorts mit der Begründung abgelehnt, sinnvolle, mess- und abgrenzbare Ziele zu finden, sei zu schwierig. Die SLB gilt meist als einfacher und weniger aufwendig. Nicht selten wird angesichts des von vielen als zu gering erachteten LOB-Volumens die SLB als Möglichkeit betrachtet, auf einen den Nutzen übersteigen(2010), Tab. 8.

6 Von dieser insgesamt positiven Bewertung ausgenommen sind die Aspekte Wirtschaftlichkeit und Personalkosteneffizienz, Effekte auf die sozialen Beziehungen und eine Begrenzung des Leistungsdrucks.
5 Zu den üblichen Bewertungskriterien vgl. auch Trittel et al. 
bereits vollzogene Personalabbau und die Übertragung zusätzlicher Aufgaben an die verbliebenen Beschäftigten, die Mehrleistung erzwingen. Beschäftigte, Personalräte und Arbeitgeber sind sich darin einig, dass die Leistungsanforderungen für die Kommunalbeschäftigten zwar merklich zugenommen haben, jedoch der leistungsorientierten Bezahlung dabei eine nachrangige Bedeutung zukommt. „Wachsende Aufgaben“, „Stellenabbau“ und „Haushaltsengpässe“ liegen als Ursachen steigender Leistungsanforderungen weit vor LOB („neue Führungsmethoden“ rangieren diesbezüglich hingegen nur wenig vor LOB).

Die beiden alternativen Methoden zur Leistungsbemessung, Zielvereinbarungen (ZV) und Systematische Leistungsbewertung (SLB), erweisen sich allerdings als unterschiedlich effektiv. ZV bewirken mehr. So geben etwa $75 \%$ der Beschäftigten mit ZV an, sie hätten „sich ernsthaft darum bemüht, ihre Ziele" zu erreichen, bei SLB bemühten sich lediglich $42 \%$ darum, durch „Leistung eine gute Bewertung zu erreichen.“ Auch nahezu alle der bereits diskutierten Positiveffekte fallen beim Einsatz von ZV stärker aus. Sowohl Arbeitgeber als auch Personalräte sind im Falle der ZV-Anwendung zufriedener mit der Einführung von LOB; etwas schwächer ausgeprägt ist dies auch bei den Beschäftigten der Fall.

Warum sind Zielvereinbarungen effektiver? ZV zwingen dazu, Leistungserwartungen in Form von Zielen zu operationalisieren, während die SLB eine rückblickende Leistungsbewertung erlaubt, ohne dass im Voraus die Leistungserwartungen hinreichend konkretisiert werden. Zwar kann auch die SLB nur dann handlungsleitend wirken, wenn Zielorientierungen für das künftige Leistungsverhalten entwickelt werden, allerdings wird die Operationalisierung der Leistungserwartungen meist den Beschäftigten überantwortet. Diese sollen leisten, wovor die Organisation sich scheut. Da die Beschäftigten jedoch meist ebenfalls damit überfordert sind, operationalisieren sie die Leistungserwartungen (der Organisation!) nicht in adäquater Weise oder sie verzichten schlicht ebenfalls darauf. Deshalb wird das Leistungsverhalten bei SLB häufig nicht oder lediglich schwach beeinflusst. Der erwartete Mangel an Objektivität der Bewertung („Nasenfaktor "), der sich in unserer Befragung bestätigt, ${ }^{7}$ ist vor allem Folgewirkung einer mangelhaften Operationalisierung von Leistungserwartungen.

Wenn kein präziser Abgleich zwischen Leistungserwartung und Leistungserbringung möglich ist, vergleichen die Beschäftigten die eigene Leistungsbewertung primär mit der ihrer Kolleginnen und Kollegen. Soweit Beschäftigte lediglich auf die Leistungsbeurteilung der anderen schielen können, verschwindet jeder Leistungsanreiz, wenn die Leistung aller oder der großen Mehrzahl der Beschäftigten positiv bewertet wird. Damit entsteht jedoch ein Dilemma: Während eine wirklich breite Prämienausschüttung die ohnehin schwachen und unpräzisen Effekte im Falle der SLB weiter unterminiert, evoziert eine stärker selektive Ausschüttung mitunter erheblichen Unmut.

Zielvereinbarungen hingegen büßen dank in der Regel klarer Operationalisierung ihre Wirkung auch dann nicht
ABB. 2

\section{Leistungsorientierte Bezahlung (LOB) - Effekte aus Beschäftigtensicht}

Angaben in Prozent, sortiert nach Mittelwert

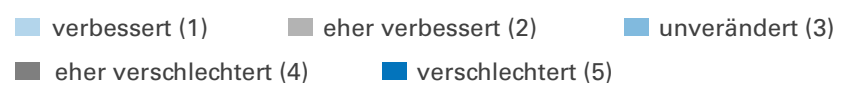

„Wie ist Ihr Eindruck: Welche Wirkungen hatte die Einführung der LOB auf nachfolgende Punkte?“*

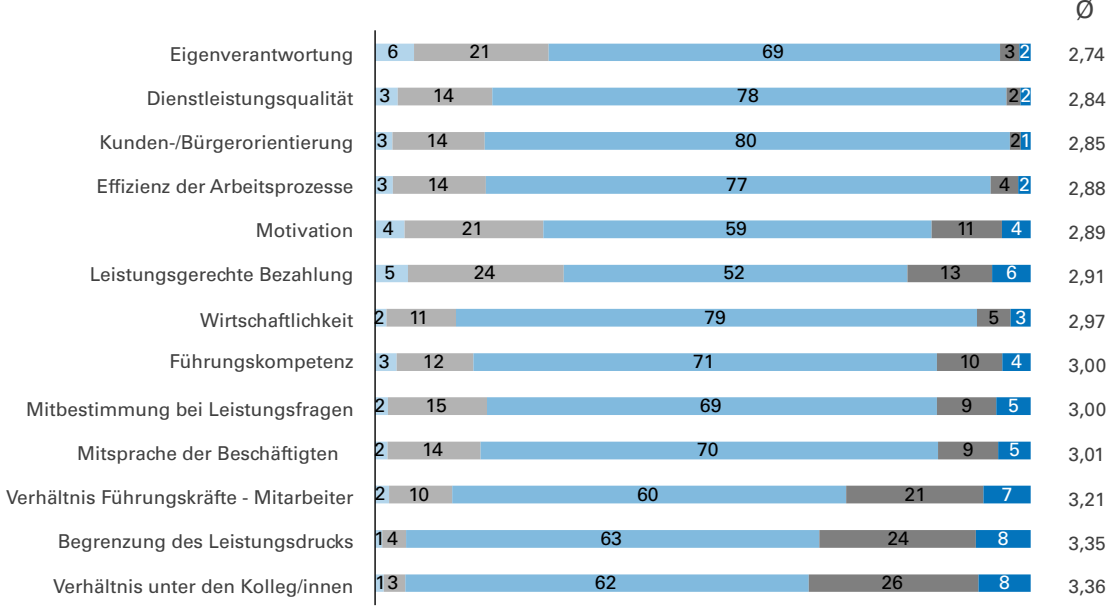

*Beschäftigtenbefragungen: Datensatz BESCH34.

Quelle: Schmidt/Müller (2013, S. 111).

ein, wenn die meisten Beschäftigten ihre Ziele erreichen. Das Verhältnis von Anreiz und Ausschüttung unterscheidet sich deshalb zwischen den Methoden grundlegend: Während Beurteilungen einen Zustand allgemein guter Leistungen, eigentlich das Ziel des Unterfangens, nicht anerkennen und somit das Tauschversprechen Leistung gegen Prämie nicht garantieren dürfen, wenn sie nicht ihre Funktionsfähigkeit verlieren wollen, können im Falle von ZV erreichbare Ziele vereinbart und deren Erreichen dann auch breit honoriert werden. Dementsprechend geben $77 \%$ der Beschäftigten mit ZV an, eine volle Prämienausschüttung erhalten zu haben, im Falle der SLB sind dies lediglich $30 \%$. Die Überlegenheit der ZV lässt sich somit sowohl aus der Principal-Agent-Perspektive als auch mittels „Cognitive Evaluation Theory“ begründen.

\subsection{Sozialbeziehungen und Partizipation}

Den schwach positiven Effekten von LOB stehen in aller Regel negative Effekte gegenüber. Häufig wird von einer Verschlechterung der sozialen Beziehungen zwischen Vorgesetzten und Mitarbeitern sowie unter den Kolleginnen und Kollegen berichtet. 60 \% der Personalräte, $54 \%$

$728 \%$ der Beschäftigten mit SLB betrachten ihre Leistung als uneingeschränkt zutreffend bewertet, während dies bei ZV $65 \%$ sind. . 
der Arbeitgeber und $70 \%$ der Beschäftigten geben an, „Neid und Konkurrenzdenken" habe zugenommen.

Negative Wirkungen auf die Sozialbeziehungen werden durch die verschiedenen Mängel bei Beurteilungsverfahren, insbesondere die meist fehlende Operationalisierung, die Intransparenz und die eingebaute Notwendigkeit zur selektiven Prämienausschüttung, befördert. So berichten 77 \% der Beschäftigten mit SLB von „Neid und Konkurrenzdenken" als Folge des Leistungsentgelts, während dies (im Vergleich nur) $55 \%$ der Beschäftigten mit ZV so sehen. Der Unterschied ist deutlich. Gleichwohl, und das macht der Wert von $55 \%$ auch mehr als anschaulich, kommen negative Effekte auf die betrieblichen Sozialbeziehungen und Unmut auch bei ZV vor.

Anders als bei SLB konstatieren im Falle von ZV sowohl Personalräte als auch Beschäftigte im Mittel leicht verbesserte Chancen der Einflussnahme auf die Leistungsbedingungen durch Personalrat oder Beschäftigte. Ver.di-nahe Personalräte berichten bei ZV zu 32 \% von einer Zunahme der Chancen der Einflussnahme (Abnahme $5 \%$ ), während dies bei SLB nur $6 \%$ tun (Abnahme $18 \%$ ). Durch eine leichte Verbesserung von Partizipations- und Mitbestimmungsmöglichkeiten werden ZV jedoch noch lange nicht zu einem Instrument der Leistungsbegrenzung, vielmehr sind sie im Unterschied zur nahezu wirkungslosen Methode SLB auch (mäßig) leistungswirksam.

\subsection{Verteilungseffekte und Diskriminierung}

In einem Teil der Kommunen tritt der erwartete Hierarchieeffekt auf. Statushöhere Fach- und Führungskräfte schneiden bei der Bewertung besser ab als Beschäftigte in den unteren Entgeltgruppen. Die Ursachen hierfür reichen von einer zweifelhaften Vermischung von Leistungsbewertung und Eingruppierung, in der höhere Fach- und Führungskräfte per se als „Leistungsträger“ gelten, über eine im Falle von wichtigen Kräften höhere Sensibilität für das Risiko der Demotivation bis zu „Korpsgeist“ und Tauschgeschäften. Ein solcher Hierarchieeffekt ist nicht nur ungerecht, sondern er delegitimiert LOB in den Augen der Beschäftigten. Meist versuchen die betrieblichen Akteure gegenzusteuern. $27 \%$ der Personalräte und $18 \%$ der Arbeitgeber geben an, Führungskräfte seien in der letzten Bewertungsrunde „besser“ bewertet worden als andere Beschäftigte. Bemerkenswert ist darüber hinaus, dass im Falle der SLB Frauen schlechter bewertet werden als Männer (35 \% der Männer und 27 \% der Frauen erhielten eine volle Prämienausschüttung). Bei ZV ist dies jedoch nicht der Fall (Vollausschüttung bei 76 \% der Männer und 78 \% der Frauen).

Stärker als von Hierarchieeffekt und „gender pay gap“ werden die Verteilungswirkungen von LOB jedoch davon geprägt, nach welchen Regeln Leistungsbewertungen in Geldbeträge übersetzt werden. In etwa $45 \%$ der Kommunen, die LOB eingeführt haben, werden bei gleicher Bewertung in Abhängigkeit von der Entgeltgruppe unterschiedliche Beträge ausgeschüttet. Ansonsten erbringt dieselbe Bewertung unabhängig von der Entgeltgruppe denselben Geldbetrag (in der gesamten Kommune oder der jeweiligen Organisationseinheit). Gegenüber dem nach Entgeltgruppen gestaffelten Tabellenentgelt ist somit trotz Hierarchieeffekt eher eine Umverteilung zugunsten der unteren Entgeltgruppen festzustellen, jedenfalls befördert LOB insgesamt betrachtet keine statusbezogene Entgeltspreizung. Beschäftigte in unteren Entgeltgruppen bewerten LOB dann auch etwas besser als andere Beschäftigte.

\section{Fazit: Akzeptanzprobleme und Änderungsbedarf}

Der Umstand, dass steigende Leistungsanforderungen im öffentlichen Dienst primär auf andere Ursachen als auf die Einführung leistungsorientierter Bezahlung (LOB) zurückzuführen sind, nimmt dem Thema die leistungspolitische Brisanz. Viele Personalräte können offenbar trotz fehlender Begeisterung nicht zuletzt deshalb mit LOB leben. Unter den Beschäftigten finden sich Gegner, Befürworter und Dulder - und alle drei Gruppen sind in ihrer Haltung ambivalent, d. h. eine grundsätzliche Zustimmung zum Leistungsprinzip geht einher mit einer ebenso grundsätzlichen Skepsis gegenüber der Leistungsbewertung. Danach gefragt, wie sie das bei ihnen „eingeführte System der leistungsorientierten Bezahlung insgesamt "beurteilen, antworten $27 \%$ der Beschäftigten mit "gut“ oder „sehr gut“, $36 \%$ mit „schlecht“ oder „sehr schlecht“" (Mittelwert einer 5er-Skala von ,sehr gut“ bis „sehr schlecht“: 3,16). Personalräte und Verwaltungsspitzen bewerten das selbst eingeführte LOB-System besser. Bei den Personalräten ergibt sich ein Mittelwert von 2,79 (PR), bei den Verwaltungsspitzen von 2,29 (AG), im Falle der Kleinkommunen von 2,61 (KL).

Viele Arbeitgeber und Personalräte stehen der LOB-Einführung eher pragmatisch gegenüber. Ihr zentraler Kritikpunkt ist nicht grundsätzlicher Natur, vielmehr sehen sie ein Missverhältnis von Aufwand und Nutzen, wobei als Aufwand meist nicht primär das auszuschüttende Budget, sondern „erhebliche Mehraufwendungen im Sinne von Transaktionskosten“ (Keller 2010, S. 106) verstanden werden. Manche Arbeitgeber und die VKA nehmen an, das Verhältnis von Aufwand und Nutzen würde sich durch ein höheres Prämienvolumen verbessern. Diese Einschätzung wird durch die in der Literatur vertretene Auffassung gestützt, dass ab einer bestimmten Höhe extrinsische Anreize den Verlust intrinsischer Motivation kompensieren können. Zunächst einmal nähme mit einer Steigerung des Budgets jedoch nicht der Nutzen, sondern der finanzielle Aufwand zu, und solange die beschriebenen Schwächen der Operationalisierung nicht behoben sind, sind wesentlich bessere Effekte unwahrscheinlich. Führungskräfte müssten mehr Zeit für LOB aufbringen, doch da viele Kommunen nicht für die zeitliche Entlastung von Vorgesetzten sorgen können, ist damit nicht zu rechnen. 
Wesentlich schlechter als das selbst eingeführte System wird von den betrieblichen Akteuren der $₫ 18$ TVöD selbst beurteilt, die tarifvertragliche Grundlage der LOB. Die Arbeitgeber (AG) bewerten diese im Mittel auf einer 5er-Skala von „sehr gut“ bis „sehr schlecht“ mit 3,0 (Arbeitgeber kleiner Kommunen 3,17) und die Personalräte mit 3,56. Mehrheitlich wird Änderungsbedarf gesehen. Lediglich ein gutes Zehntel der Arbeitgeber sowie $6 \%$ der Personalräte sehen keinerlei Änderungsbedarf.

Es besteht jedoch keine Einigkeit darüber, was verändert werden soll. $56 \%$ der Arbeitgeber (AG) sprechen sich für eine weitere Erhöhung des Budgetvolumens aus. Personalräte stimmen dem zu 47 \% zu. Anders die Arbeitgeber kleiner Kommunen: Sie vertreten diese Position mehrheitlich nicht. Zugleich sind $55 \%$ der Personalräte dafür, den $\$ 18$ TVöD abzuschaffen und das Entgeltvolumen pauschal auszuschütten (bei Wegfall des Volumens nur $21 \%$ ). Viele Personalräte lehnen Leistungsentgelt zwar nicht prinzipiell ab, jedoch die in $\$ 18$ getroffenen Regelungen. Mehrheitlich sind die Personalräte somit für die Abschaffung des $\$ 18$ (soweit das Entgeltvolumen nicht verloren geht), ersatzweise wäre für viele aber auch eine Ausweitung des Volumens akzeptabel. Eine solche Position mag zunächst wenig konsistent erscheinen, sie passt jedoch ganz gut zur Ambivalenz der Beschäftigten. Eine gemeinsame Tendenz findet sich lediglich bei der Forderung nach Freiwilligkeit der LOB. Jeweils absolute Mehrheiten der Personalräte, der Arbeitgeber und der Arbeitgeber kleiner Kommunen sind sich einig, dass die LOB-Einführung für kleine Kommunen optional sein sollte. Relative Mehrheiten von 46 \% (AG), 50 \% (PR) und 46 \% (KL) sprechen sich auch dafür aus, die Einführung allen Kommunen freizustellen.

Mit dem $₫ 18$ TVöD wurde offenbar eine Regel geschaffen, die erstens eine ausgesprochen heterogene Umsetzung erlaubt: Die Praxis reicht - das zeigen unsere Analysen - von der Nicht-Einführung der LOB (und zwar in beträchtlichem Ausmaß) über eine defizitäre Umsetzungspraxis (in vielen Fällen) bis zu eher anspruchsvollen Zielvereinbarungskonzepten (in der Minderheit der untersuchten Fälle). Zweitens hat der $\$ 18$ TVöD höchst heterogene Interessenorientierungen der Akteure hervorgebracht: Zwar wird von nahezu allen „Betroffenen“ Änderungsbedarf gesehen bzw. angemahnt, doch kaum eine Veränderung würde von einer breiten Mehrheit getragen werden. Gleichwohl: Die Tarifparteien sollten über Veränderungen nachdenken, auch wenn der Status quo sich als zumindest weniger dramatisch erweist als im Vorfeld der LOB-Einführung vielfach angenommen. Aber ein befriedigendes Ergebnis wurde auch nicht erzielt, da viel Aufwand für wenig Nutzen getrieben werden muss. Akzeptable Resultate könnten sein: die optio-nale Anwendung von Leistungsentgelt, ein koordinierter Rückbau, eine qualitative Verbesserung oder eine auch eine im Beschäftigteninteresse liegende sinnvolle anderweitige Verwendung der Mittel. Den ungeteilten Zuspruch der Beschäftigten würde jedoch, wie bereits erwähnt, keine dieser Veränderungen finden, da bei den Beschäftigten Leistungsbewertung zwar auf wenig Begeisterung stößt, viele die Prämie jedoch durchaus zu schätzen wissen. Immerhin $36 \%$ der Beschäftigten stimmen dem Statement „LOB ist ein gute Sache“ $\mathrm{zu}, 37 \%$ Zustimmung findet aber auch die Aussage „Ich bin gegen LOB“. Bei einer Abschaffung des Leistungsentgelts müsste es gelingen, den Beschäftigten zu vermitteln, dass diese ohne Entgeltverlust erfolgt. Dies dürfte insbesondere deshalb nicht einfach sein, da damit zu rechnen ist, dass die VKA - würde sie sich je darauf einlassen - einen Verzicht auf LOB mit Einspareffekten legitimieren würde.

Die eingangs aufgeworfene Frage, ob Leistungsentgelt im öffentlichen Dienst prinzipiell funktionsfähig ist, ist folglich differenziert zu beantworten: Durch eine auf qualitative Verbesserungen zielende und beteiligungsorientiert angelegte Anwendung von Zielvereinbarungen können durchaus Effekte erzielt werden. Leistungsentgeltsysteme, die allerdings ausschließlich auf materielle Anreize, unilaterale Beurteilungsverfahren und Mehrleistung setzen, versprechen im öffentlichen Dienst aufgrund der „passiven Stärke“ der Beschäftigten sowie der spezifischen Tätigkeits- und Motivationsstrukturen keinen Erfolg. Voraussetzung für eine gelingende LOB-Umsetzung ist es jedoch, dass Personalräte und Beschäftigte dafür gewonnen werden. Ohne einen tragfähigen Konsens der Tarifparteien kann dies zumindest im deutschen öffentlichen Dienst kaum gelingen. Es dürfte der eigentliche Geburtsfehler der LOB-Einführung gewesen sein, dass die tarifliche Vereinbarung von Leistungsentgelt nicht Ausdruck eines gemeinsamen Willens der Tarifparteien ist, sondern einem gewerkschaftlichen „concession bargaining“ geschuldet war, das zur Rettung des Flächentarifvertrages unumgänglich schien. Da eine nachträgliche Herstellung eines solchen Konsenses kaum realisierbar sein dürfte, muss die Einführung von leistungsorientierter Bezahlung im deutschen öffentlichen Dienst in der Fläche als weitgehend gescheitert gelten.

\section{LITERATUR}

Agrawal, Ch. (2012): Performance-related pay - hype versus reality. With special reference to public sector organizations, in: Management and Labour Studies 37 (4), S. 337-344

Bahnmüller, R./Schmidt, W. (2009): Riskante Modernisierung des Tarifsystems. Die Reform der Entgeltrahmenabkommen am Beispiel der Metall- und Elektroindustrie Baden-Württembergs, Berlin

Breisig, T. (1998): Personalbeurteilung - Mitarbeitergespräch - Zielvereinbarungen. Grundlagen, Gestaltungsmöglichkeiten und Umsetzung in Betriebsund Dienstvereinbarungen, Frankfurt a. M.

Breisig, T. (2003): Entgelt nach Leistung und Erfolg. Grundlagen moderner Entlohnungssysteme, Frankfurt a. M.

Brown, W./Marginson, P./Walsh, J. (2003): The management of pay as the influence of collective bargaining diminishes, in: Edwards, P. (Hrsg.): Industrial relations, theory and practice, Oxford, S. 189-213

Dannenberg, O. (2013): Undifferenziertes Leistungsentgelt bei fehlender Dienstvereinbarung, in: Der Personalrat 30 (3), S. 98-101

Deci, E. L. (1971): Effects of externally mediated rewards on intrinsic motivation, in: Journal of Personality and Social Psychology 18 (1), S. 105-115

Deci, E. L. (1976): The hidden costs of rewards, in: Organizational Dynamics 4 (3), S. $61-72$

Demmke, Ch. (2009): Leistungsbezahlung in den öffentlichen Diensten der EUMitgliedstaaten - Eine Reformbaustelle, in: dms - Zeitschrift für Public Policy, Recht und Management 4 (1), S. 53-71

Dürk, B./Sternatz, R. (2007): Leistungsentgelt - Stichwort: Zielvereinbarungen. Chancen eines umstrittenen Instruments, in: Der Personalrat 24 (10),

S. 418-442

Fischer, H. (2010): Vorrang Tarifvertrag. Die Regulierung variabler Entgeltsysteme bei der Deutschen Telekom Geschäftskunden (DTGK), in: Sterkel, G./Ganser, P./Wiedemuth, J. (Hrsg.): Leistungspolitik: neu denken. Erfahrungen Stellschrauben - Strategien, Hamburg, S. 60-92

Frey, B. S. (1997): On the relationship between intrinsic and extrinsic work motivation, in: International Journal of Industrial Organization 15 (4), S. 427-439 Frey, B. S./Jegen, R. (2001): Motivation Crowding Theory, in: Journal of Economic Surveys 15 (5), S. 589-611

Gesamtmetall (2013): Die deutsche Metall- und Elektro-Industrie in der Bundesrepublik Deutschland in Zahlen, Berlin

Herbing, Th. (2004): Leistungsbemessung, Beteiligung und Motivation - Perspektiven für die gewerkschaftliche Politik, in: Kuhlmann, S./Bogumil, J./Wollmann, H. (Hrsg.): Leistungsmessung und -vergleich in Politik und Verwaltung. Konzepte und Praxis, Wiesbaden, S. 361-373

Keller, B. (2010): Arbeitspolitik im öffentlichen Dienst. Ein Überblick über Arbeitsmärkte und Arbeitsbeziehungen, Berlin

Kessler, I./Purcell, J. (1992): Performance related pay: objectives and application, in: Human Resource Management Journal 2 (3), S. 16-33 
Kratzer, N./Nies, S. (2009): Neue Leistungspolitik bei Angestellten. ERA, Leistungssteuerung, Leistungsentgelt, Berlin

Lazear, E. P./Gibbs, M. (2009): Personnel economics in practice, Hoboken, NJ Marsden, D. (2010): The paradox of performance-related pay systems: Why do we keep adopting them in the face of evidence that they fail to motivate?, in: Margetts, H./6, P./Hood, C. (Hrsg.): Paradoxes of modernization. Unintended consequences of public policy reform, Oxford, S. 185-202

Matiaske, W./Weller, I. (2008): Leistungsorientierte Vergütung im öffentlichen Sektor. Ein Test der Motivationsverdrängungsthese, in: Zeitschrift für Betriebswirtschaft (ZfB) 78 (1), S. 35-60

Organisation for Economic Co-operation and Development (OECD) (Hrsg.)

(2005): Performance-related pay policies for government employees, OECD Publications, Paris

Perry, J. L. (1996): Measuring public service motivation: an assessment of construct reliability and validity, in: Journal of Public Administration Research and Theory 6 (1), S. 5-22

Perry, J. L./Wise, L. R. (1990): The motivational bases of public service, in: Public Administration Review 50 (3), S. 367-373

Ryan, R. M./Deci, E. L. (2000): Intrinsic and extrinsic motivations: classic definitions and new directions, in: Contemporary Educational Psychology 25 (1), S. 54-67

Sadowski, D./Pull. K./Schneider, M. (1999): Vertrauen: Voraussetzung oder Ergebnis effizienter Arbeitsbeziehungen? - Gutenbergs Solidaritätsaxiom und die institutionenökonomische Unternehmenstheorie, in: Albach, H./Eymann, E./Luhmer, A./Steven, M. (Hrsg.): DieTheorie der Unternehmung in Forschung und Praxis, Berlin/Heidelberg/New York, S. 537-549

Sansone, C./Harackiewicz, J. M. (Hrsg.) (2000): Intrinsic and extrinsic motivation. The search for optimal motivation and performance, San Diego, CA Schettgen, P. (1996): Arbeit - Leistung - Lohn. Analyse- und Bewertungsmethoden aus sozioökonomischer Perspektive, Stuttgart

Schmidt, W./Müller, A. (2013): Leistungsorientierte Bezahlung in den Kommunen. Befunde einer bundesweiten Untersuchung, Reihe Modernisierung des öffentlichen Sektors, Sonderband 42, Berlin

Schmidt, W./Müller, A./Trittel, N. (2011a): Der Konflikt um dieTarifreform des öffentlichen Dienstes. Verhandlungsprozesse und Umsetzungspraxis, Reihe Modernisierung des öffentlichen Sektors, Sonderband 38, Berlin
Schmidt, W./Müller, A./Trittel, N. (2011b): Leistungsentgelt im öffentlichen Dienst: Intentionen, Wirkungen und Akzeptanz, in: Industrielle Beziehungen $18(1-2)$, S. $78-98$

Tondorf, K. (2007): Tarifliche Leistungsentgelte - Chance oder Bürde, Reihe Modernisierung des öffentlichen Sektors, Bd. 29, Berlin

Tondorf, K./Jochmann-Döll, A. (2004): Monetäre Leistungsanreize im öffentlichen Sektor, in: WSI-Mitteilungen 57 (8), S. 428-434, http://www.boeckler.de/ wsimit_2004_08_tondorf.pdf

Tondorf, K./Jochmann-Döll, A. (2005): (Geschlechter-)Gerechte Leistungsvergütung? Vom (Durch-)Bruch des Leistungsprinzips in der Entlohnung, Hamburg

Trittel, N./Schmidt, W./Müller, A./Meyer, T. (2010): Leistungsentgelt in den Kommunen. Typologie und Analyse von Dienst- und Betriebsvereinbarungen. Reihe Modernisierung des öffentlichen Sektors, Sonderband 35, Berlin

\section{AUTOREN}

WERNER SCHMIDT, Dr., ist Wissenschaftler und Geschäftsführer des Tübinger Forschungsinstituts für Arbeit, Technik und Kultur (F.A.T.K.). Arbeitsschwerpunkte: Arbeits- und Industriesoziologie, Industrielle Beziehungen, Arbeitsbeziehungen des öffentlichen Dienstes, Migrationsforschung.

@w.schmidt@uni-tuebingen.de

ANDREA MÜLLER, M.A., ist Wissenschaftlerin am F.A.T.K. in Tübingen. Arbeitsschwerpunkte: Arbeitssoziologie, Arbeitsbeziehungen des öffentlichen Dienstes in Deutschland und Europa.

and.mueller@uni-tuebingen.de 